evidence for benefit and the risk of serious harm from accidental retention, routine insertion of throat packs should be questioned and the risk/benefit ratio discussed for each individual patient at the theatre team brief.

\section{A PROFILE OF THE PSYCHOSOCIAL IMPACT OF CHRONIC, ORGANIC VOICE DISORDERS IN THE ADOLESCENT POPULATION AND THE FACTORS THAT AFFECT THIS}

${ }^{1}$ Ashton Brower, ${ }^{1}$ Lesley Cavalli, ${ }^{2}$ Nicola Gorb. ${ }^{1}$ Great Ormond Street Hospital and University College London; ${ }^{2}$ Great Ormond Street Hospital

10.1136/archdischild-2020-gosh.19

Clinical voice disorders in children arise from, or are associated with, both congenital and acquired laryngeal conditions and whilst many can be managed to a point of resolution some disorders may be expected to havea life-long impact on vocal function. Research indicating the intractable nature of some laryngeal disorders is relatively recent (Reynolds, Meldrum, Simmer, Vijayasekaran, \& French, 2017), and studies exploring the nature of impact arising from chronic dysphonia in young people are sparse.

There is currently no clear profile of the chronically voice disordered adolescent population nor reported evidence as to those factors that may increase the risk of negative impact arising from a chronic voice disorder.

Our study evaluated whether the perceived impact of chronic organic dysphonia, primarily of structural and neurological aetiology, changes across adolescence and aimed to identify other factors such as gender, vocal aetiology, vocal tract discomfort, perceptual voice features, and voice severity, that might impact trends.

Data was collected retrospectively from the health records of 67 subjects from the historic voice caseload of a Speech and Language Therapy Service within a tertiary children's hospital. Subjects ranged from $10-18$ years and their data extracted from the first recorded contact. Regression analyses were used to evaluate the impact of age and other factors on self-perceived psychosocial impact, measured by the Voice Handicap Index.

Results indicate that age can predict self-perceived psychosocial impact of chronic organic dysphonia for the majority of adolescents. The factors of gender, vocal tract discomfort and asthenic voice quality further impact self-perceived psychosocial impact. Interestingly, overall perceptual voice severity was not a predictor of perceived psychosocial impact and in itself should therefore not be used to guide decisions around the need for intervention. Further research is much needed in this field.

\section{DEVELOPMENT OF A DIGITAL GUIDE FOR FAMILIES OF PATIENTS ON THE GOSH PAEDIATRIC INTENSIVE CARE UNIT}

${ }^{1} Y u$ Du, ${ }^{1}$ Yuexi Fang, ${ }^{1}$ Kamile Sakelyte, ${ }^{2}$ Daiana Bassi, ${ }^{2}$ Sue Conner, ${ }^{1} Y u n$ Fu, ${ }^{1}$ Dean Mohamedally, ${ }^{2}$ Gemma Molyneux, ${ }^{3}$ Graham Roberts, ${ }^{2}$ Neil J Sebire, ${ }^{4}$ Rufaro Ndokera, ${ }^{4}$ Samiran Ray. ${ }^{1}$ UCL Department of Computer Sciences; ${ }^{2} D R I V E$, Great Ormond Street Hospital for Children NHS Foundation Trust; ${ }^{3} U C L ;{ }^{4} P I C U$, Great Ormond Street Hospital for Children NHS Foundation Trust

10.1136/archdischild-2020-gosh.20
Paediatric Intensive Care Units (PICUs) can be very stressful environments for families of sick children. PICU has more restrictions than general wards, and activities such as visiting hours can be very different from other areas of the hospital. Currently, information is available to families in paper form and displayed around the unit. However, we would like to revolutionise our communication strategy to make information available digitally in the form of a mobile app, allowing families to engage with this information more flexibly.

As part of a joint collaboration between GOSH and UCL computer science (CS) through the industry exchange network, a mobile app that delivers information to parents was developed using Ionic. A web application, which allows clinical teams to manage content, was developed using NodeJS with a MySQL database.

The mobile app delivers a broad range of information about the PICU ward, including information on access, staff groups, and equipment used. In addition, a FAQ section is included that was developed in partnership with patients and families. The submission of Feedback is also possible via the app; this allows users to submit questions, or comments, on the content and information. This mobile app is an example of how the Hospital might develop new ways of communicating important information to patients and families using our services.

\section{DEVELOPMENT OF A DIGITAL SELF-CARE MANAGEMENT APPLICATION FOR CHILDREN WITH UVEITIS: UVEITIS PATIENT PASSPORT}

${ }^{1}$ Elizaveta Kretova, ${ }^{1}$ Asrath Rahman, ${ }^{2}$ Daiana Bassi, ${ }^{1} Y u n$ Fu, ${ }^{1}$ Dean Mohamedally,

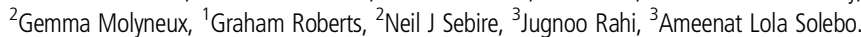
${ }^{1}$ UCL Department of Computer Sciences; ${ }^{2}$ DRIVE, Great Ormond Street Hospital for Children NHS Foundation Trust, ${ }^{3}$ UCL Great Ormond Street Institute of Child Health

\subsection{6/archdischild-2020-gosh.21}

Childhood uveitis is a chronic, sight threatening inflammatory eye condition. There are a complex range of different disorders within the collective term of uveitis. Children can have associated systemic inflammatory disorders, and are treated with systemic chemotherapies which need frequent monitoring. For many, the uveitis continues to be active in adulthood. Care for children with uveitis is multi-centre and multi-disciplinary. A child's primary care team may be located in a geographically distant location, acting as an obstacle to informing teams at local hospitals, particularly when children present acutely with problems associated with their eye disease, systemic disease or treatment.

It can also be a challenge to educate young people about their disease, which is vital to support transition. A paper based 'Uveitis Passport' existed to support self-care for adults with uveitis. This paper based personal record was well received, but was not designed for use in children and young people. We have undertaken a project to create a digital mobile app specifically aimed at children to support them, and their families, to take control of their condition.

In collaboration with UCL computer science, through the industry exchange network, researchers at GOSH and UCL GOS Institute of Child Health developed a mobile app to support Uveitis self-management. The adult 'passport' content was modified with the support of children and families. The 